\title{
Corrigendum
}

\section{Corrigendum to "Impact of Diabetic Ketoacidosis on Thyroid Function in Patients with Diabetes Mellitus"}

\author{
Yuling Xing $\mathbb{D}^{1,2}$ Jinhu Chen $\mathbb{D}^{1},{ }^{1}$ Guangyao Song $\mathbb{D}^{1,3,4}$ Liying Zhao $\mathbb{D}^{1,2}$ \\ and Huijuan $M a \mathbb{D}^{1,3,4}$ \\ ${ }^{1}$ Department of Endocrinology, Hebei General Hospital, Shijiazhuang 050017, China \\ ${ }^{2}$ Graduate School of Hebei Medical University, Shijiazhuang 050017, China \\ ${ }^{3}$ Hebei Key Laboratory of Metabolic Diseases, Hebei General Hospital, Shijiazhuang 050051, Hebei, China \\ ${ }^{4}$ Department of Internal Medicine, Hebei Medical University, Shijiazhuang 050017, Hebei, China
}

Correspondence should be addressed to Huijuan Ma; huijuanma19@163.com

Received 8 May 2021; Accepted 8 May 2021; Published 26 May 2021

Copyright (C) 2021 Yuling Xing et al. This is an open access article distributed under the Creative Commons Attribution License, which permits unrestricted use, distribution, and reproduction in any medium, provided the original work is properly cited.

In the article titled "Impact of Diabetic Ketoacidosis on Thyroid Function in Patients with Diabetes Mellitus" [1], the authors identified an error in the presentation of the flowchart in Figure 1, and the corrected figure is as follows:

The authors and the editorial board agree to the publication of the corrigendum.

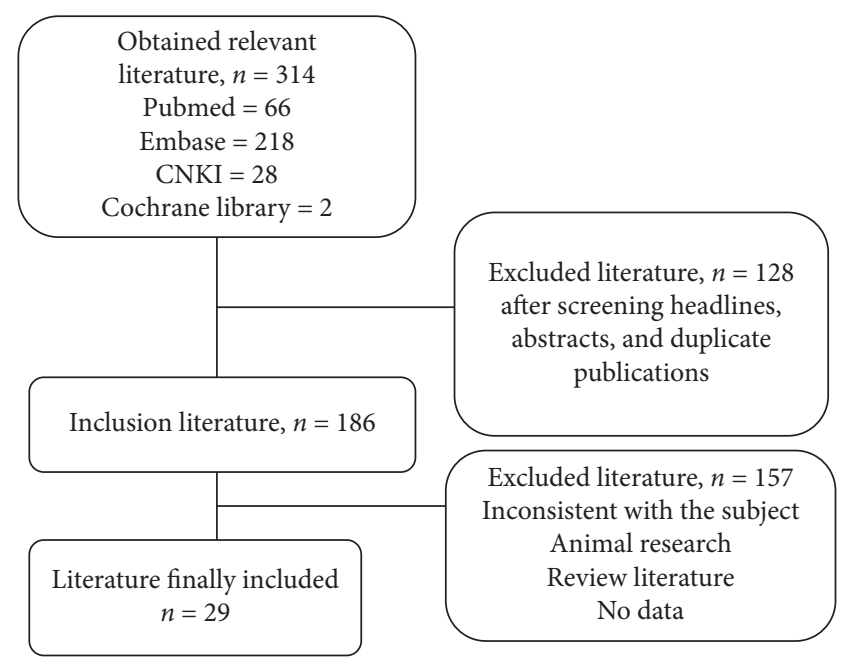

FIGURE 1: The process of study selection.

\section{References}

[1] Y. Xing, J. Chen, G. Song, L. Zhao, and H. Ma, "Impact of Diabetic Ketoacidosis on Thyroid Function in Patients with Diabetes Mellitus," International Journal of Endocrinology, vol. 2021, Article ID 2421091, 26 pages, 2021. 